\title{
MEDICAL SCENARIO AT HIGH ENVIRONMENTAL TEMPERATURE
}

\author{
Muhammad Aslam and Ghulam Mustafa Lodhi
}

Pakistan is blessed with a diversity of weather conditions ranging from extreme cold to extreme hot weathers that may be accompanied by humidity. The relationship between human health and stressful weather is a multifaceted medical, social and environmental issue. Heat related illnesses are known since Biblical times. ${ }^{1}$ The scientific reports on heat related casualties date back to 1743, when 11000 persons died in China due to heat wave in one month. Slower march of climate alterations present a formidable challenge for health professionals and the humanity en bloc. Increasing evidence shows that atmospheric carbon dioxide levels are at rise and causing global warming. Global warming indicators suggest that average earth temperature has risen between $0.4^{\circ} \mathrm{C}$ and $0.8^{\circ} \mathrm{C}$ over last century and envisaged increase in temperatures by year 2100 is between $1.5^{\circ} \mathrm{C}$ and $5.8^{\circ} \mathrm{C}$. As a result of this, heat waves creating stressful weather with appalling consequences are projected to increase in sternness and frequency. The number of people killed annually by weather disasters between 1972 and 1996 was about 123000 and most of them were in Africa and Asia. ${ }^{2}$

Health hazards of extreme temperatures frequently ensue, if people are subjected to temperatures with whom they are not acclimatized. Thermoregulation in humans is a mandatory requirement to maintain homeostasis. Rise of temperature less than $1^{\circ} \mathrm{C}$ activates peripheral and hypothalamic heat receptors that signal hypothalamic thermoregulatory centre. Resultant physiological response to heat entails increasing cutaneous circulation thereby promoting heat loss through radiation, convection, perspiration and evaporative cooling.

Successive increments in level of work performed in hot and humid climates acclimatize the person to perform in unendurable and life threatening heat. Acclimatization may be achieved in weeks and involves enhancement of cardiovascular performance, activation of renin-angiotensin-aldosterone axis, salt conservation by renal and non-renal mechanisms, expansion of plasma volume, increase in GFR and ability to resist exertional rhabdomyolysis. When body's thermoregulatory responses are inadequate to preserve homeostasis, heat illness ensues that may range from mild muscle cramps and sunburns to life threatening heat strokes.

Heat exhaustion is a milder entity that exists on the same continuum of heat illness. It may present with non-specific signs and symptoms like mild pyrexia and circulatory collapse, associated with hyponatremia and dehydration resulting in hypohydrosis with consequent thermoregulatory failure. On the other hand, heat stroke is a serious and potentially life threatening illness considered as a form of hyperthermia due to systemic inflammatory response leading to multiorgan dysfunction syndrome with predominant encephalopathy. Sequence of events proposed in pathophysiology of heat stroke is complex. Heat stress induces thermoregulatory, acute phase and heat shock responses. Thermoregulation involves active sympathetic cutaneous vasodilation, elevated blood temperature induced tachycardia with resultant increase in cardiac output and increased minute ventilation. Also reduced visceral perfusion and enhanced cutaneous circulation facilitate heat dissipation. However, dehydration, salt depletion and cardiovascular diseases may blight thermoregulation.

Acute phase response is a coordinated reaction involving endothelial cells, leukocytes and epithelial cells that protect against tissue injury and promote repair. Cytokines produced in response to heat stress mediate hyperpyrexia, leukocytosis, acute phase proteins anabolism, muscle catabolism and stimulation of hypothalamo-pituitary-adrenal axis. Heat stress induced production of interleukin- 6 modulates systemic and local acute inflammatory responses controlling the levels of inflammatory cytokines. Interleukin-6 also stimulates hepatic production of anti-inflammatory acute phase proteins which are responsible for inhibition of reactive oxygen species production, release of proteolytic enzymes from leukocytes and may also stimulate endothelial cells adhesion, proliferation and angiogenesis thus playing a role in healing and repair. Heat shock response comprises production of heat shock proteins at cellular level. Production of heat shock proteins is regulated at the level of genetic transcription, the transcription factors being activated by heat stress. Attenuation of heat shock response during heat stress is suggestive of its protective nature. Heat shock proteins may function as molecular chaperones that bind to partially folded or unfolded proteins, preventing their irreversible denaturation. They are also thought to act as baroreceptor reflex regulator in heat stress and confer CVS protection by abating hypotension and bradycardia. Thus, these proteins 
improvise the cell endurance by inducing tolerance to heat stress. If expression levels less due to aging, lack of acclimatization or certain genetic polymorphisms then case of heat stress may advance hastily to heat stroke.

Local and systemic insults resulting from heat stress, such as splanchnic hypoperfusion alter the immunological and barrier functions of intestines. This results in leakage of endotoxins, increased production of inflammatory cytokines that induce endothelial cell activation and release of endothelial vasoactive factors like nitric oxide and endothelins. The inflammatory and coagulation response to heat stress along with direct heat induced cytotoxic effects result in injury to vascular endothelium and microthrombosis. Both pyrogenic cytokines and endothelium derived factors can interfere with normal thermoregulation by elevating the set point at which sweating is galvanized. Also there is alteration in vascular tone particularly in splanchnic circulation, which precipitates hypotension, hyperthermia and heat stroke. Disseminated intravascular coagulation, cardiac arrhythmias, hepatorenal failure, neurological involvement, encephalopathy and coma may further worsen the prognosis.,

Regarding management of heat stroke cases, along with prompt and effective cooling measures, new concepts are emerging. Salicylates and non-steroidal antiinflammatory drugs activate heat shock transcription factors and induce the transcription and translation of heat shock proteins which may enhance the tolerance to heat and cellular protection against heat stress. Animal studies have shown that immunomodulators like interleukin-1 receptor antagonists, antibodies to endotoxins and corticosteroids improve survival in heat stroke cases. Therapy with recombinant activated protein $\mathrm{C}$ and tissue factor pathway inhibitors may also provide promising results in heat strokes.
Perpetually confronted with dry and humid environmental temperatures across the country, preventive measures against heat stress are of considerate significance. Reliable meteorological forecasts for warning against oncoming heat waves can serve a lot. Working hours for outdoor labourers, unacclimatized soldiers and marathon runners need to be readjusted, like exertional activities in early morning and evening with mid day siesta. Adequately maintained hydration status with electrolyte balance also contributes towards prevention of heat stroke.

As the intimidation to heat illness is increasing, recognition of the facts that thermoregulatory failure and impaired regulation of inflammatory and stress responses contribute to tissue injury and facilitate progression towards heat stroke should make research in this direction a priority. Better knowledge of body's responses to heat stress at cell and molecular level will help towards novel preventive measures and a new paradigm of immunomodulation. It will definitely provide the heat stroke induced multiorgan dysfunction cases a better management and prevention, thus catastrophes from the natural calamity may be reduced.

\section{REFERENCES}

1. Contenau G. Everyday life in Babylon and Assyria. New York, Saint Martin's Press; 1954.

2. Lorette A, Tegegen Y. Disasters in Africa: old and new hazards and growing vulnerability. World Health Stat Q. 1996; 49: 179-84.

3. Bouchama A, Knochel JP. Heat stroke. N Engl J Med. 2002; 346: 1978-88.

4. Glazer JL. Management of heat stroke and heat exhaustion. Am Family Physician. 2005; 71: 213340.

\begin{tabular}{|l|}
\hline AUTHOR AFFILIATION: \\
Maj. Gen. Muhammad Aslam, HI (M) (Corresponding Author) \\
Professor and Head, \\
Department of Physiology, \\
Army Medical College, \\
Rawalpindi - Pakistan. \\
Email: maslam@nust.edu.pk
\end{tabular}

